\title{
Quality- Of- Survey Routing Solutions forMobile Ad HocNetworks: A Review
}

\author{
Dr MadhumitaDash ${ }^{1}$, Mrs Ricky Mohanty ${ }^{2}$ \\ ${ }^{1}$ (Dept. Of ETC ,OEC, India, \\ ${ }^{2}$ (Dept. Of ETC , OEC, India ,
}

\begin{abstract}
The field of mobile ad hoc networks (MANETs), is still in its infancy and particularly the provision of quality of service $(Q \circ S)$ is much more challenging comparing to the wired line networks, mainly due to node mobility, multihop communications, contention for channel access, and a lack of central coordination. Now a days much research attention has focused on providing QoS assurances in MANET protocols. QoS routing protocols provide a mechanism to establish a multicast session. The QoS routing protocol is an integral part of any QoS solution since its function is to ascertain which nodes, if any, are able to serve applications requirements and also is an open problem and remains relatively uncharted territory. This paper offers a survey of QoS routing solutions for MANETs which requires the interaction and cooperation of several components like QoS routing protocol, resource reservation scheme and QoS capable medium access control (MAC) layer. This paper provides a broad and comprehensive view of the various components and protocols required to provide QoS support in computer networks, focusing primarily on ad hoc networks which shows the picture of transform of traditional infrastructure wired to wireless networks (i.e. cellular-based networks).In this paper, an overview of QoS multicast protocolswhich have been proposed in the past literatures are presented. In addition, the performancesof these protocols are compared with respect to performance metrics.
\end{abstract}

Keywords: mobile ad hoc networks (MANETs), quality of service (QoS), QoS-base medium access control

\section{Introduction}

Mobile Ad-hoc Network (MANET) is a wireless system that comprises mobile nodes. It is usually referred to a decentralized autonomous system. Mobile nodes engaged in MANET often work as client/servers. Nodes in the network can be either fixed or mobile. Mobile nodes include laptop, mobile phone, MP3 player, home computer or personal digital assistance. Nodes may be located on ships, airplanes or land, irrespective of their location as they can participate in communication. Self connectivity and easy deployment of MANETs makes it apt for emergency, surveillance situations and rescue operations. The field of mobile ad hoc networks (MANETs) [3] has been recognized as an area of research for more than ten years due to their decentralized, self-configuring, and dynamic nature, which avoids the need for an expensive base station infrastructure. The emergence of real-time applications and the widespread use of wireless and mobile devices have generated the need to provide quality-of-service (QoS) support in wireless and mobile networking environments.QoS provision in MANETs is a challengingtask since in addition to obeying QoS constraints wemust account also for a dynamic topology and sharedwireless medium. The aim of these protocols was to provide a basic best-effort level of service to ensure network operation in the face of an unpredictable and shared wireless communicationmedium and to maintain a network topology view and routes in the face of failing links and mobile devices.Current wireless networks support mobile/wireless access for mobile communications devices by providing a wireless interface between the mobile devices and a fixed network of limited range base-stations (BS). On the basis of this infrastructuremodel for wireless communications, the air-interface consists of a single data-link terminating on a BS. Communication from that point is routed across a fixed network to its destination. Mobility is managed by allocating a limited set of communications frequency channels to each BS, and dynamically assigning a mobile device to a local channel as it moves from the coverage area of one BS to another. While providing QoS in an infrastructure environment is difficult, supporting QoS in mobile ad hoc networks, which do not dependon a BS for communications, is even more difficult.Essentially, a mobile ad hoc network is a networkof mobile routers. Properties Of Mobile ad hoc Networks: ad hoc networks are selforganizing, rapidly deployable and require no fixed infrastructure [1-3] and are composed of wireless mobile nodes (e.g. a router consisting of multiple hosts and equipped with wireless communication capability) that can be deployed anywhere, and must cooperate in order to dynamically establish communications using limited network management and administration [4]. 


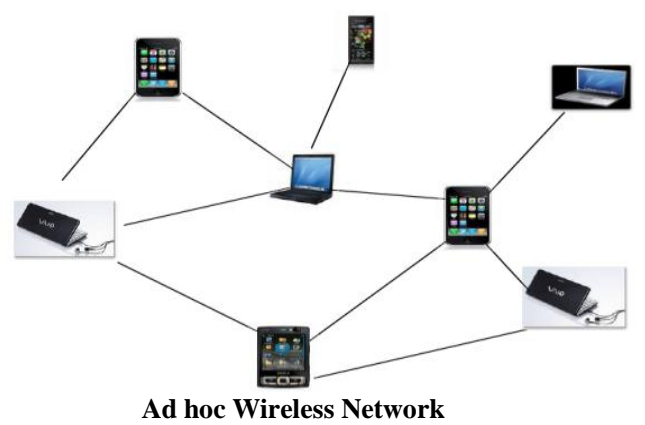

Nodes in an ad hocnetwork may be highly mobile, or stationary, and mayvary widely in terms of their capabilities and uses [4,5]. They may operate autonomously or connected tothe Internet.Environments in which ad hoc networks are initiallyexpected to play an important role includeinstant infrastructure scenarios, particularly wheremobile access to a wired network is either ineffectiveor impossible. Because of their inherent flexibility, adhoc networks have the potential to serve as a ubiquitouswireless infrastructure capable of interconnectingthousands of devices [6], and supporting a wide rangeof networking applications. It is hoped that in thefuture, ad hoc networks will emerge as an effectivecomplement to wired or wireless LANs, and even towide-area mobile networking services, such as PersonalCommunication Systems (PCS). In order toachieve this status, however, applications and servicesequivalent to those available in these environmentsmust be made available to ad hoc network users.

Challenges Of Mobile ad hoc Networks:

1. There is no centralized authority for network control,routing or administration (e.g. BS).

2. The network topology may change randomlyand rapidly and may consist of both bidirectionaland unidirectional links.

3. It is difficult to determine theaggregate bandwidth between two endpoints.

4. There is a difficult task for best-effort routing without even considering QoS requirements.

5. Mobile nodes that are end points for user communications and applications must act cooperatively to handle network functions, mostly notably routing and MAC, without specialized routers.

\section{II.Quality -Of-Service: Definition and Overview}

According to RFC2386 [7], QoS is a set of service requirements to be met by the network while transporting a flow. A flow is a packet stream from a source to a destination (unicast or multicast) with an associated (Q o S). The associated QoS could, in fact, be 'best effort'. A fundamental requirement of any QoS mechanism is a measurable performance metric. Typical QoS metrics include available bandwidth, packet loss rate, estimated delay, packet jitter, hop count and path reliability. Analogous to today's Internet, ad hoc networks are being designed to provide best-effort service (i.e. do not provide any guarantees regarding packet loss or delay, available bandwidth, jitter etc.). In a best-effort service model, packets are dropped regardless of their importance. If a packet is lost, the sender can simply retransmit the lost packet. This method is efficient for applications that do not require bounds on packet delay or other QoS metrics. However, real-time applications, such as video-on-demand ( $\mathrm{VoD}$ ), videoconferencing and Internet telephony have, are sensitive to packet loss and delay and may have minimum bandwidth requirements Consequently, the best-effort service may not be suitable for these applications. Technically, there are two ways in which QoS can be achieved: (1) overprovisioning and (2) traffic engineering. Overprovisioning utilizes the best-effort approach and simply increases the available resources (e.g. bandwidth, buffers etc.). For example, network designers could simply increase the capacity of a congestion link or network from 10 to $100 \mathrm{Mb}$. Generally, a QoS model does not define specific protocols or implementations. Instead, it defines the methodology and architecture by which certain types of services (e.g. per-flow or class-based) can be provided in the network.

\section{III.QoS Routing In Mobile Ad Hoc Networks}

QoS routing protocols search for that routes in which sufficient resources are present to satisfy QoS requirements. These protocols work with the resource management mechanisms to establish the paths through the network that meet the end-to-end QoS requirements, such as delay and bandwidth demand. QoS routing is difficult in MANETs because overheads is too high and the bandwidth is limited so there must be a mechanisms for a mobile node to store and update link information. Dynamic nature of MANETs, make it extremely difficult for obtaining accurate link information. The reserved resource may not be guaranteed because high mobility of node causes path breakage. QoS routing is an essential part of the QoS architecture. Before any connections can be made or any resources reserved, a feasible path between a source destination pair must be established. QoS routing is a routing mechanism under which paths for flows are determined on the basis of some knowledge of 
resource availability in the network as well as the QoS requirements of the flows or connections [21]. The signaling mechanism can be operated independent of the routing protocol. There is no interaction between the QoS resource reservation mechanism and the routing protocol. The routing protocol only provides the best route between the source and destination. The signaling protocol establishes resources along the route chosen by the routing protocol. The routing protocol provides a route between the source and destination of the QoS flow. The Q oS signaling feedback to the routing protocol regarding the route chosen and asks the routing protocol for alternate routes if the route provided, doesn't satisfy the Q o S requirements. The objectives of QoS routing are threefold:

(1) if one exists, find a feasible path between a source destination pair (i.e. a path that has sufficient available resources capable of satisfying the QoS requirements),

(2) optimize the use of network throughput and network resources and

(3) adapt to network congestion, providing smooth performance degradation to lower-priority traffic.

The QoS routing protocol tries to find a path that has a good chance of meeting the QoS requirements [21].

Designers of QoS routing algorithms for ad hoc networks must consider several design issues:

(1) metric selection (e.g. bandwidth, delay etc.)and path computation, (2) QoS state propagation

and maintenance and (3) scalability. The QoS routing protocol must also deal with imprecise state information due to node (router) movement and topology changes. Furthermore, a QoS routing scheme for ad hoc networks must balance efficiency and adaptively, while maintaining low-control overhead. Several protocols are proposed in the literature with a provision of QoS at the level of routing. Their objectives and aims differ based on their applications and strategies used, one of them is

\section{Bandwidth estimation based routing}

Generally, routing protocols find a feasible route from a source to a destination without taking into account current traffic in the network or specific requirements of an application. As a result, the network may become overloaded and the requirements of a real-time application that requires a support for QoS may not be met. Therefore, there seems a need to estimate the traffic in the network so that a feedback can be provided to the application in case when its requirements cannot be met. A QoS aware routing that is based on the bandwidth estimation for mobile ad hoc networks is proposed in Chen and Heinzelman (2005). The protocol incorporates an admission control scheme together with a feedback scheme to meet the QoS requirements of real-time applications. The QoS routing protocol is based on Ad hoc On-demand Distance Vector (AODV) routing. The protocol is based on the intuition that the end-to-end throughput of a route depends upon the minimum end-toend residual bandwidth available along the route. To estimate available residual bandwidth, two methods are used. In the first method, hosts listen to the channel and estimate the available bandwidth that is based on the ratio of the time for which the channel is free and the time for which the channel is busy. This estimate is called 'listen' bandwidth estimation. In the second method, every host disseminates the information about the bandwidth used by it currently. This dissemination is carried out through 'hello' messages. A host estimates its available bandwidth indicated in the 'hello' messages that it receives from its two hop neighbours. This is called the 'hello' bandwidth estimation. The performance of 'hello' bandwidth estimation comes out to be better as compared to the 'listen' bandwidth estimation when releasing the bandwidth is required immediately. However, 'listen' bandwidth estimation does not incur an additional overhead as compared to 'hello' bandwidth estimation. The reason is that in 'hello' bandwidth estimation, the information about bandwidth consumed by neighbours is attached in 'hello' messages, and these 'hello' messages are sent periodically.

A QoS routing protocol called Bandwidth Reservation in Ad hoc Wireless Networks (BRAWN) is proposed in Guimaraes et al. (2009). BRAWN is also based on bandwidth estimation, however, it is designed for multirate networks. In a multirate network, nodes can choose among several modulation schemes and can communicate to neighbours using different transmission rates depending on channel conditions. BRAWN provides a network layer solution i.e., no modification is required at the lower layers. The scheme employed computes the available bandwidth at a node which is then used to accept or reject a flow.

\section{Issues and Challenges Providing QoS In Ad-Hoc Networks}

QoS provision will lead to an increase in computational and communicational cost. In other words, it requires more time to setup a connection and maintains more state information per connection. The improvement in network utilization counterbalances the increase in state information and the associated complexity and various issues are needed to be faced while providing QoS for MANETS. The major problems that are faced are as follows:

Unreliable wireless channel: the wireless channel is prone to bit errors due to interference from othertransmissions, thermal noise, shadowing and multi-path fading effects [7]. This makes it impossible to providehard packet delivery ratio or link longevity guarantees. 
Node mobility: the nodes in a MANET may move completely independently and randomly as far as thecommunications protocols are concerned. This means that topology information has a limited lifetime andmust be updated frequently to allow data packets to be routed to their destinations. Again, this invalidates anyhard packet delivery ratio or link stability guarantees. Furthermore, QoS state which is link- or node position dependentmust be updated with a frequency that increases with node mobility. An important general assumption must also be stated here: for any routing protocol to be able to function properly, the rate of topology change must not be greaterthan the rate of state information propagation. Otherwise, the routing information will always be stale and routing will be inefficient or could even fail completely. This applies equally to QoS state and QoS route information.A network that satisfies this condition is said to be combinatorially stable [3].

Lack of centralized control: the major advantage of an ad hoc network is that it may be set up spontaneously, without planning and its members can change dynamically. This makes it difficult to provide any form of centralized control. As such, communications protocols which utilize only locally-available state and operate ina completely distributed manner, are preferred [8]. This generally increases an algorithm's overhead and complexity, as QoS state information must be disseminated efficiently.

Channel contention: In order to discover network topology, nodes in a MANET must communicate on acommon channel. However, this introduces the problems of interference and channel contention. For peer-topeerdata communications these can be avoided in various ways. One way is to attempt global clock synchronizationand use a TDMA-based system where each node may transmit at a predefined time. This is difficult toachieve due to the lack of a central controller, node mobility and the complexity and overhead involved [9].Other ways are to use a different frequency band or spreading code (as in CDMA) for each transmitter.This requires a distributed channel selection mechanism as well as the dissemination of channel information.However data communications take place, without a central controller, some set-up, new neighbor discoveryand control operations must take place on a common contended channel. Indeed, avoiding the aforementioned complications, much MANET research, as well as the currently most popular wireless ad hoc networking technology $(802.11 \mathrm{x})$ is based on fully-contended access to a common channel i.e. with CarrierSense Multiple Access with Collision Avoidance (CSMA/CA). However, CSMA/CA greatly complicates the calculation of potential throughput and packet delay, compared to TDMA-based approaches. This is because nodes must also take into account the traffic at all nodes within their carrier sensing range. Furthermore, the possibility of collisions also arises. Collisions waste channel capacity, as well as node battery energy, increase delay, and can degrade the packet delivery ratio. Finally, the well-understood hidden node [10] andexposed node [11] problems are a further consequence of channel contention. These problems are even more pronounced when we consider that nodes may interfere with transmissions outside of their transmission range [12], [9], [13], since receivers are able to detect a signal at a much greater distance than that which they can decode its information.

Limited device resources: to some extent this is an historical limitation, since mobile devices are becomingincreasingly powerful and capable. However, it still holds true that such devices generally have less computational power, less memory and a limited (battery) power supply, compared to devices such as desktop computers typically employed in wired networks. This factor has a major impact on the provision of QoS assurances, since low memory capacity limits the amount of QoS state that can be stored, necessitating more frequent updates, which incur greater overhead. Additionally, QoS routing generally incurs a greater overhead than best-effort routing in the _rst place, due to the extra information being disseminated. These factors lead to a higher drain on mobile nodes' limited battery power supply. Finally, within the pool of QoS routing problems, many are NP-complete [3], and thus complicated heuristics are required for solving them, which may place an undue strain on mobile nodes' less-powerful processors.

\section{Protocol Classification}

In [5], QoS routing protocols are classified chiefly by their:

- Treatment of network topology (flat, hierarchical, or location-aware)

- Approach to route discovery (proactive, reactive, hybrid, or predictive) On the other hand, in [6] they are classified in three different ways, based upon

- The interaction between the route discovery and QoS provisioning mechanism (coupled or decoupled)• The interaction with the MAC layer (either independent or dependent)

- Again, on the approach to route discovery.

In general, depending upon at which layer of TCP/IP protocol suite the QoS provisioning is implemented, one can classify methods of QoS provisioning into the following categories:

- MAC layer

- network layer

- cross layer. 


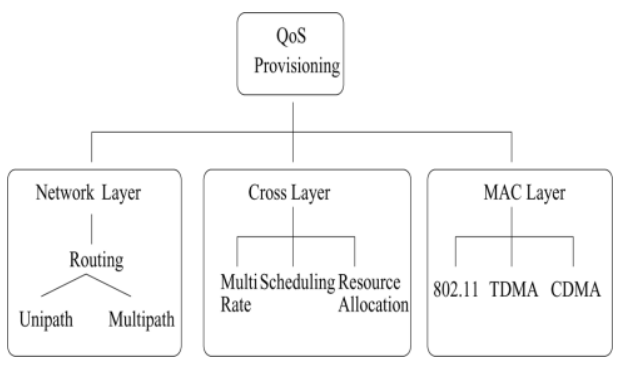

\section{A layered classification of methods of QoS provisioning in ad hoc networks}

Based on the methodologies used, these categories can be further divided into subcategories. For example, MAC layer schemes are divided broadly into three subcategories:

- IEEE 802.11

-TDMA

- CDMA.

There can be several other MAC layer protocols as well. Cross layer strategies are also divided into three subcategories, namely, scheduling, multirate, and resource allocation. The strategies at the network layer consists mainly of routing protocols which can be divided into unipath and multipath categories.

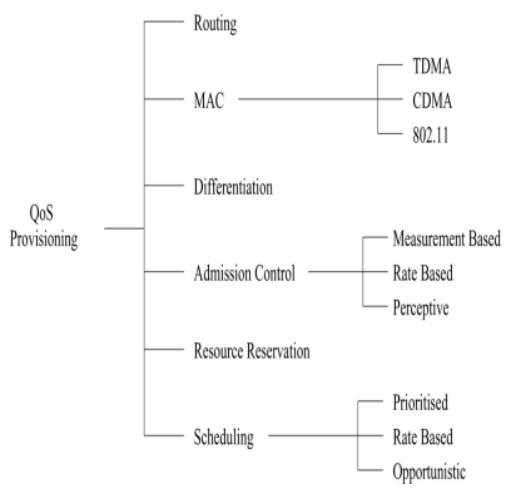

VI. QoS Architectures

In general, an architectural framework consists of a group of modules that are required for the QoS provisioning in an ad hoc network e.g., routing, MAC layer, admission control, resource reservation, etc. Some of the architectural frameworksproposed in the literature for the provision of QoS in mobile ad hoc networks. An IP-based framework, called INSIGNIA,3 for providing QoS in mobile ad hoc networks is presented in Lee et al. (2000). It uses in-band signaling. The term in-band signalingmeans that the control information is carried with data, and there is no separate control channel as opposed to another type of signaling called out-of-band signaling where control information and data are sent on separate channels. The framework is aimed to provide adaptive QoS guarantees to an application. By adaptive, we mean that there is a minimum QoS that has to be provided to an application. The level of QoS can be enhanced later if resources required to support the enhanced QoS are available. The architecture has several modules that are routing,in-band signaling, admission control, packet forwarding or scheduling, MAC protocol, etc. However, it is a stateful architecture because it uses soft state resource management scheme to utilize the resources.

\section{A. Metrics used to specify QoS requirements}

The following is a sample of the metrics commonly used by applications to specify QoS requirements to the routing protocol. Consequently, they may be used as constraints on route discovery and selection. Each metricis followed by a reference which provides an example ofa protocol that employs the metric as a QoS constraint._ Minimum required throughput or capacity (bps) -the desired application data throughput. The former incorporatesthe queuing delay at each node and thelatter is determined by the propagation delay andthe transmission time of a packet. The transmissiontime between two nodes is simply the packet sizein bits / the channel capacity.

\section{B. Node states and metrics employed for route selection}


This section lists many of the metrics commonlyemployed by routing protocols for path evaluation andselection in order to improve all-round QoS or to meet the specific requirements of application data sessions.Many of these metrics, especially those measured atlower layers, are not directly interesting to the applicationlayer, hence their listing in this section. However, they all, at least indirectly, affect the QoS experienced by a data session.

1) Network Layer Metrics:

Achievable throughput or residual capacity (bps) The achievable data throughput of a path or node.The achievable throughput or residual capacity isoften termed available bandwidth. in the literature; we prefer to reserve the use of the word .bandwidth

. for quantifying the size of frequency bandsin $\mathrm{Hz}$.

2) Link and MAC Layer Metrics:

MAC delay the time taken to transmit a packetbetween two nodes in a contention-based MAC,including the total time deferred and the time toacknowledge the data [22]. This provides a goodindication of the amount of traffic at the relevantnodes; Link reliability or frame delivery ratio (\%) thestatistically calculated chance of a packet or framebeing transmitted over a link and correctly decodedat the receiver. See [23], [24] for examples of routing protocols employing this metric for pathselection. Node relative mobility/stability can be measuredas the ratio of the number of neighbours that change over axed period to the number that remainthe same [25]. For example, if all of the node'sneighbors are the same over axed period, thatnode is completely stable in that period, relative to its neighbors.

3) Physical Layer Metrics:

Signal-to-interference ratio (SIR) - although a physicallayer metric, the received SIR at a destinationnode can be used as a routing metric that shows linkquality, via cross-layer communication. Example ofuse: [26]; Bit error rate (BER) related closely to SIR, thisvalue determines the level of error correction and/ornumber of retransmissions required over a .link.and has a major impact on the link's reliabilitymetric and on energy consumption. From anotherperspective, the BER is a consequence of the SIRbetween two nodes. For an example of use, see [27]; Node residual battery charge or cost [20]. Examplesof use: [28], [23];Finally, path reliabilityis a multiplicative metric, since the reliabilities of eachlink in the path must be multiplied together to computethe chance of delivering the packet via a given route(assuming that the MAC layer retransmissions have beenconsidered in the reliability value, or that there are noretransmissions e.g. for broadcast packets).

\section{VII.Protocol Evaluation Metrics}

The following metrics may be used to evaluate a QoS routing protocol's performance.

\section{1) Transport/Application Layer:}

Session acceptance/blocking ratio the percentageof application data sessions (or transport layer connections)that are admitted into or rejected fromthe network. The value of this metric rejects boththe effectiveness of the QoS protocols as well asconditions outside of their control, such as channelquality;Session completion/dropping ratio, this metric representsthe percentage of applications that were successfully/unsuccessfully served after being admittedto the network. For example, if a VoIP session isaccepted and the session is completed properly (bythe users hanging up) and not aborted (dropped) dueto route failure or any other error, then that countsas a completed session.

2) Network Layer:

Network throughput (bps) - the amount of datatraffic the entire network carried to its destination in one second; Per-node throughput (bps) - the average throughputachieved by a single node; ${ }_{-}$Route discovery delay (s) (for reactive protocols) -a measure of the effectiveness of reactive protocols,i.e. on average, what is the delay between a routerequest being issued and a reply with a valid routebeing received. In some cases, this may also bereferred to as the session establishment time (SET),Normalized routing load (NRL) - the ratio of routing.

3) MAC Layer:

Normalized MAC load - similar to the NRL, this represents the ratio of bits sent as MAC control frames to the bits of user data frames transmitted. Example of use: [29]; MAC energy efficiency - ratio of energy used forsending data bits to the total energy expended fordata plus MAC headers and control frames

\section{Factors Affecting QoS Protocol Performance}

When evaluating the performance of QoS protocols, a number of factors has a major impact on the results.Some of these parameters are particular manifestationof characteristics of the MANET environment. They define the scenario whether in simulation or real-life, and can be summarized as follows:

Node mobility - this factor generally encompassesseveral parameters: the nodes' maximum and minimumspeed, speed pattern and pause time. Thenode's speed pattern determines whether the nodemoves at uniform speed at all times or whether itis constantly varying, and also how it accelerates, for example uniformly or exponentially with time.The pause time determines the length of time nodesremain stationary between each period of 
movement.Together with maximum and minimum speed,this parameter determines how often the networktopology changes and thus how often network stateinformation must be updated. This parameter hasbeen the focus of many studies; Network size - since QoS state has to be gathered ordisseminated in some way for routing decisions tobe made, the larger the network, the more difficultthis becomes in terms of update latency and messageoverhead. This is the same as with all networkstate information, such as that used in besteffort protocols [8]; Number, type and data rate of traffic sources - intuitively,a smaller number of traffic sources results infewer routes being required and vice-versa. Trafficsources can be constant bit rate (CBR) or maygenerate bits or packets at a rate that varies withtime according to the Poisson distribution, or anyother mathematical model. The maximum data rate affects the number of packets in the network.

\section{QoS Guided Route Discovery}

In an on-demand ad hoc network routing protocol, such as DSR or AODV, a node (which we call the initiator) can find a route to a destination node (which we call the target) by per-forming a controlled flood of the network. In this Route Discovery procedure, the initiator transmits a ROUTE REQUEST packet, identifying the target to which the route is needed. Each node receiving the ROUTE REQUEST in general re-transmits the REQUEST if it has not already forwarded a copy of it; when the target node receives the REQUEST, it returns a ROUTE REPLY to the initiator, listing the route taken by the REQUEST, rather than forwarding the REQUEST. Many optimizations have been defined for this basic Route Discovery scheme to reduce the frequency of performing Route Discovery and to limit the portion of the network over which the ROUTE REQUEST flood must be forwarded.

\section{Conclusion}

In this article we reviewed the challenges to and basic concepts behind QoS routing in MANETs and provided a thorough overview of QoS routing metrics and design considerations where protocols were selected in such a wayas to highlight many different approaches to QoS routing in MANETs, while simultaneously covering most of the importantadvances in the field. We summarized the operation, strengths, anddrawbacks of these protocols in order to enunciate the varietyof approaches proposed and to expose the trends in designersthinking. The protocols' interactions with the MAC layer werealso described. Finally, we provided an overview of the area sand trends of progress in the field and identified topics for future research .The provision of QoS in an ad hoc network is a challenging task and the challenge comes due to inherent characteristics of such a network. In this paper, we presented an overview of the research related to the provision of QoS in mobile ad hoc networks. The contributions of the paper are as follows.

- issues and challenges in providing the QoS in a mobile ad hoc network. Some of thesechallenges are due to the typical nature of theproblem and others are due to inherentcharacteristics and limitations associated with anad hoc network.

- classifications of themethodologies used to provide QoS in mobile ad hoc networks. The first classification called layeredclassification is based on the layer of the protocolstack to which the corresponding methodologybelongs. The second classification called functionalclassification is based on the functionality providedby the corresponding methodology.

- discussed various methodologies reported in the literature that provide QoS in one form or theother and pointed out salient features of eachmethodology and compared methodologies thataddress somewhat similar issues.

- After describing the methodologies proposed in theliterature, we pointed out issues that may beaddressed in future for almost all majorfunctionalities that are necessary for the provision of QoS in an ad hoc network.

\section{References}

[1]. Quality of service provisioning in ad hoc wireless networks: a survey of issues and solutions..available online:http://www.sciencedirect.com, Apr 2004.

[2]. Alwan A, Bagrodia R, Bambos N, Gerla M, KleinrockL,Short J, Villasenor J. Adaptive Mobile Multimedia Networks. IEEE Personal Communications Magazine 1996; 3(2): 34-51.

[3]. Ahn G-S, Campbell AT, Lee S-B, Zhang X. INSIGNIA. Internet Draft, draft-ietf-manet-insignia-01.txt, October 1999.

[4]. D. Kim, “A New Mobile Environment: Mobile Ad Hoc Networks (MANET)," IEEE Vehic. Tech. Soc. News, Aug. 2003, pp. 29-35.

[5]. S. Chen, Routing Support for Providing Guaranteed End-to-End Quality-of-Service, Ph.D. thesis, University of Illionois at UrbanaChampaign, 1999.

[6]. S. Chakrabarti and A. Mishra, "QoS Issues in Ad Hoc Wireless Networks," IEEE Commun. Mag., vol. 39, Feb. 2001, pp. 142-48.

[7]. S. Chakrabarti and A. Mishra, "Quality of Service Challenges for Wireless Mobile Ad Hoc Networks," Wiley J. Wireless Commun. and Mobile Comp., vol. 4, pp. 129-53, Mar 2004.

[8]. J. N. Al-Karaki and A. E. Kamal, "Quality of Service Routing in Mobile Ad Hoc Networks: Current and Future Trends," Mobile Computing Handbook, I. Mahgoub and M. IIays, Eds., CRC Publishers, 2004.

[9]. T. B. Reddy et al., "Quality of Service Provisioning in Ad Hoc Wireless Networks: A Survey of Issues and Solutions," April 2004, available online: http://www.sciencedirect.com

[10]. S. Saunders, Antennas and Propagation for Wireless Communication Systems Concept and Design, New York: Wiley, 1999. 
[11]. C. E. Perkins, Ed., Ad Hoc Networking, Ch. 3., Addison Wesley, 2001.

[12]. Y. Yang and R. Kravets, "Contention-Aware Admission Control for Ad Hoc Networks," IEEE Trans. Mobile Comp., vol. 4, Aug. 2005, pp. 363-77.

[13]. L. Kleinrock and F. Tobagi, "Packet Switching in Radio Channels, Part II: The Hidden Terminal Problem in Carrier Sense Multiple-Access Modes and the Busy-Tone Solution," IEEE Trans. Commun., vol. 23, no. 12, 1975, pp. $1417-33$.

[14]. D. Shukla, L. Chandran-Wadia, and S. Iyer, "Mitigating the Exposed Node Problem in IEEE 802.11 Ad Hoc Networks," Proc. 12th Int'l. Conf. Computer Commun. and Networks, Oct.2003, pp. 157-62.

[15]. Y. Yang and R. Kravets, "Contention-Aware Admission Control for Ad Hoc Networks," tech. rep., University of Illinois at Urbana Champaign, 2003.

[16]. L. Chen and W. Heinzelman, "QoS-Aware Routing Based on Bandwidth Estimation for Mobile Ad Hoc Networks," IEEE JSAC, vol. 23, Mar. 2005, pp. 561-72.

[17]. C. R. Lin and J.-S. Liu, "QoS Routing in Ad Hoc Wireless Networks," IEEE JSAC, vol. 17, Aug. 1999, pp. $1426-38$.

[18]. S. Saunders, Antennas and Propagation for Wireless Communication Systems Concept and Design. New York, USA: JohnWiley and Sons, 1999.

[19]. C. E. Perkins, ed., Ad Hoc Networking, ch. 3. Addison Wesley,2001.

[20]. Y. Yang and R. Kravets, .Contention-aware admission control forad hoc networks,.IEEE Trans. Mobile Comput., vol. 4, pp. 363.377, Aug 2005.

[21]. L. Kleinrock and F. Tobagi, .Packet switching in radio channels part II: The hidden terminal problem in carrier sense multipleaccessmodes and the busy-tone solution,.IEEE Trans. Commun., vol. 23, no. 12, pp. 1417.1433, 1975.

[22]. D. Shukla, L. Chandran-Wadia, and S. Iyer, .Mitigating the exposed node problem in IEEE 802.11 ad hoc networks,. InProc. 12th Int. Conf. Computer Communications and Networks,pp. 157.162, Oct. 2003.

[23]. Y. Yang and R. Kravets, .Contention-aware admission control for ad hoc networks,. tech. rep., University of Illinois at UrbanaChampaign, 2003.

[24]. L. Chen and W. Heinzelman, .QoS-aware routing based on bandwidth estimation for mobile ad hoc networks,.IEEE J.Select. Areas Commun., vol. 23, pp. 561.572, Mar. 2005.

[25]. C. R. Lin and J.-S. Liu, .Qos routing in ad hoc wireless networks,.IEEE J. Select. Areas Commun., vol. 17, pp. 1426.1438, Aug. 1999 .

[26]. S. Chen and K. Nahrstedt, .Distributed quality-of-service routing in ad hoc networks,.IEEE J. Select. Areas Commun., vol. 17 pp. 1488.1505, Aug. 1999.

[27]. A. R. Bashandy, E. K. P. Chong, and A. Ghafoor, .Generalized quality-of-service routing with resource allocation,.IEEE J.Select. Areas Commun., vol. 23, pp. 450.463, Feb 2005.

[28]. M. Wang and G.-S. Kuo, .An application-aware QoS routing scheme with improved stability for multimedia applications inmobile ad hoc networks,.inProc. IEEE Vehicular Technology Conf., pp. 1901.1905, Sep. 2005.

[29]. A. Abdrabou and W. Zhuang, .A position-based qos routing scheme for UWB mobile ad hoc networks,.IEEE J. Select. AreasCommun., vol. 24, pp. 850.856, Apr. 2006.

[30]. M. Sheng, J. Li, and Y. Shi, .Routing protocol with QoS guarantees for ad-hoc network,.Electronics Letters, vol. 39,pp. 143.145, Jan. 2003.

[31]. S. Singh, M. Woo, and C. S. Raghavendra, .Power-aware routing in mobile ad hoc networks,.inProc. Int. Conf. Mobile Computingand Networking, pp. 181.190, 1998.

[32]. I. Rubin and Y.-C. Liu, .Link stability models for QoS ad hoc routing algorithms,. in Proc. 58th IEEE Vehicular Technology Conf., vol. 5, pp. 3084.3088, Oct. 2003.

[33]. Z. Fan, QoS routing using lower layer information in ad hoc networks,.inProc. Personal, Indoor and Mobile Radio CommunicationsConf., pp. 135.139, Sep. 2004.

[34]. A. Misra and S. Banerjee, .MRPC: Maximising network lifetime for reliable routing in wireless environments,.inProc.IEEE Wireless Communications and Networking Conf., (Orlando,Florida), March 2002.

[35]. Wu, D. and Negi, R. (2005) 'Utilizing multiuser diversity for efficient support of quality of service over a fading channel', IEEE Transactions on Vehicular Technology, Vol. 54, No. 3, May, pp.1198-1206.

[36]. Wu, H. and Jia, X. (2007) 'QoS multicast routing by using multiple paths/trees in wireless ad hoc networks', ElsevierJournal on Ad hoc Networks, Vol. 5, No. 5, July,pp.600-612.

[37]. Xiao, H., Seah, K.G., Lo, A. and Chua, K.C. (2000) 'A flexible quality of service model for mobile ad hoc networks', Proc.Fifty First IEEE Vehicular Technology Conference IEEE Press, Tokyo, Vol. 1, pp.445-449.

[38]. Xiao, Y. and Li, H. (2004) 'Local data control and admission control for QoS support in wireless ad hoc networks',IEEE Transactions on Wireless Communications, Vol. 53,No. 5, September, pp.1558-1568.

[39]. Xiao, Y. (2005) 'Performance analysis of priority schemes for IEEE 802.11 and IEEE 802.11e wireless LANs', IEEETransactions on Wireless Communications, Vol. 4, No. 4,July, pp.1506-1515.

[40]. Xu, Y. and Guerin, R. (2005) 'Individual QoS versus aggregate QoS: a loss performance study', IEEE/ACM Transactionson Networking, Vol. 13, No. 2, April, pp.370-383.

[41]. Xu, J. and Lipton, R.J. (2005) 'On fundamental tradeoffs between delay bounds and computational complexity in packet scheduling algorithms', IEEE/ACM Transactions on Networking, Vol. 13, No. 1, February, pp.15-28.

[42]. Xue, G., Sen, A., Zhang, W., Tang, J. and Thulasiraman, K. (2007) 'Finding a path subject to many additive QoS constraints', IEEE/ACM Transactions on Networking, Vol. 15, No. 1, February, pp.201-211.

[43]. Yang, Y. and Kravets, R. (2005) 'Contention-aware admission control for ad hoc networks', IEEE Transactions on Mobile Computing, Vol. 4, No. 4, August, pp.850-856.

[44]. Yang, Y. and Kravets, R. (2007) 'Throughput guarantees for multipriority traffic in ad hoc networks', Elsevier Journalon Ad Hoc Networks, Vol. 5, pp.228-253.

[45]. Zhai, H., Chen, X. and Fang, Y. (2005) 'How well can the IEEE 802.11 wireless LAN support quality of service?',IEEE Transactions on Wireless Communications, Vol. 4,No. 6, November, pp.3084-3094.

[46]. Zhang, B. and Mouftah, H.T. (2005) 'QoS routing for wireless ad hoc networks: problems, algorithms, and protocols',IEEE Communications Magazine, October, pp.110-117.

[47]. Zhao, Q. and Tsang, D.H.K. (2006) 'An equal-spacing-based design for QoS guarantee in IEEE 802.11e HCCA wirelessnetworks', IEEE Transactions on Mobile Computing, Vol. 7, No. 12, December, pp.1491-1503. 